\title{
Commentary
}

\section{Deception of Subjects in Neuroscience: An Ethical Analysis}

\author{
Franklin G. Miller ${ }^{1}$ and Ted J. Kaptchuk ${ }^{2}$ \\ ${ }^{1}$ Department of Bioethics, National Institutes of Health, Bethesda, Maryland 20892-1156, and 2Massachusetts General Hospital/Massachusetts Institute of \\ Technology/Harvard Medical School Athinoula A. Martinos Center for Biomedical Imaging and Osher Research Center, Harvard Medical School, Boston, \\ Massachusetts 02215
}

Deception is a common feature of research design in neuroscience, which is adopted to promote scientific validity. Nevertheless, it is ethically controversial because it compromises informed consent. Scientific articles publishing the results of deceptive research typically do not explicitly mention that deception was used and include misleading statements that informed consent was obtained from the research participants. In this article, we illustrate this lack of transparency regarding deception in neuroscience with respect to three recent examples of research on, or related to, the placebo effect. We recommend two procedures for promoting the autonomy of research participants in studies involving deception as well as publication guidelines for scientific journals relating to deceptive research.

Deception of research subjects typically involves deliberately misleading communication by investigators about the purpose of the research and the nature of experimental procedures. Such deception is used when it is thought that truthful disclosure to subjects may influence the very phenomena under investigation. For some important scientific questions-especially concerning human perception, sensation, interpretation, behaviors, and attitudes-if subjects are fully informed of the hypothesis or research design, the experiment might be unable to

Received April 7, 2008; accepted April 9, 2008.

T.J.K. was supported by Grants P01 AT002048 and 1K24 AT004095 from the National Center for Complementary and Alternative Medicine at the National Institutes of Health (NIH). The opinions expressed in this report are solely those of the authors and do not reflect the position or policy of the National Institutes of Health, the Public Health Service, or the Department of Health and Human Services. We thank David Wendler for helpful comments on a previous draft of this paper.

Correspondence should be addressed to Dr. Franklin G. Miller, Department of Bioethics, Clinical Center, National Institutes of Health, Building 10, Room 1C118, Bethesda, MD 20892-1156. E-mail: fmiller@nih.gov.

D0I:10.1523/JNEUROSCI.1493-08.2008

Copyright $\odot 2008$ Society for Neuroscience $\quad$ 0270-6474/08/284841-03\$15.00/0 provide valid knowledge. For example, informing subjects that the object of study is visual processes that are not the subject of conscious attention might change the visual processes being studied. Or investigating how different amounts of monetary reward or peer pressure influence decisions or neural networks cannot be easily undertaken if the study goal is declared. In some circumstances, transparent informed consent can produce bias and jeopardize scientific knowledge. Hence, there are often sound methodological reasons to adopt deception or the absence of full disclosure to promote the internal and external validity of the research (Korn, 1997).

Despite this scientific rationale, deception in the experimental design of human subjects research is ethically controversial (Wendler and Miller, 2004). Nevertheless, it remains common in psychological research and is also frequently used in neuroscience. The major ethical problem with deception in human experimentation is that it runs contrary to the basic principle of respect for persons and the related requirement of investigators to obtain informed consent for research participation (National Commission for the Protection of Human Subjects of Biomedical and Behavioral Research, 1979). Given that human research must be reviewed and approved by research ethics committees before being implemented and that informed consent is a basic ethical and regulatory requirement, how is it possible for deceptive research to be conducted? In the United States, the federal regulations governing human subjects research permit Institutional Review Boards (IRBs) "to approve a consent procedure which does not include, or which alters, some or all of the elements of informed consent ... or to waive the requirement to obtain informed consent" provided that four conditions are satisfied: "(1) the research involves no more than minimal risk to the subjects; (2) the waiver or alteration will not adversely affect the rights and welfare of the subject; (3) the research could not practicably be performed without the waiver or alteration; and (4) whenever appropriate, the subjects will be provided with additional pertinent information after participation" (Department of Health and $\mathrm{Hu}-$ man Services, 1991).

Perhaps because of the ethical cloud covering deceptive research, published articles reporting the results of deceptive experiments typically have not been transparent about the use of deception. This lack of transparency is reflected in two pervasive characteristics of the scientific literature reporting deceptive experimentation. First, descriptions of research methods typically do not highlight the use of deception. Instead, the use of deception must be inferred by the reader. Those who are not alert to the possibility that deception may be used, or lack sensitivity to ethical concerns that deception raises, may fail to notice the ways in which experiments are deceptive. More problematic is the typical boilerplate statement in published articles of deceptive studies that informed consent was obtained from research participants. These points are illustrated below with respect to three published neuroscience articles reporting the results of research on, or related to, the placebo effect. After describing the lack of transparency about deception in these examples, we discuss two procedures for promoting autonomy of research participants in studies involving deception, and the role of scientific journals in setting publication guidelines for the reporting of such research.

\section{Deceptive neuroscience research}

The placebo effect has long been a source of scientific interest and concern 
(Kaptchuk, 1998). On the one hand, making use of the placebo effect has the potential to enhance therapeutic outcomes in clinical medicine. On the other hand, the placebo effect is a potential source of bias in clinical trials that complicates the evaluation of treatment efficacy. Investigating whether and how the expectations, attitudes, and previous experiences of human subjects may substantially influence their response to medical treatment and to clinical encounters opens up a fascinating window into the relationship between mind and body, with important theoretical and practical implications (Guess et al., 2002). With the development of brain imaging technology, neuroscientists have devoted increasing attention to probing the neurobiology of the placebo effect and related influences of expectation in the context of controlled experimentation (Benedetti et al., 2005). Yet because such placebo experimentation depends on eliciting strong expectations that subjects are receiving a pharmacologically or physically active intervention, when in fact they are receiving an "inert" placebo (Vase et al., 2002; Charron et al., 2006), these experiments usually deceive subjects regarding the nature of the treatment they are administered, as well the purpose of the research. Three studies selected from many with similar forms of deception are described below with respect to the way deception is addressed in the published report.

A recent neuroscience research report on the brain network involved with placebo analgesia, to which one of us (T.J.K.) contributed, displays a lack of full transparency regarding the use of deception (Kong et al., 2006). Healthy volunteers were recruited to investigate brain functioning relating to expectation effects using placebo acupuncture, a thermal pain stimulus, and functional magnetic resonance imaging (fMRI). But subjects were told something else; they were informed about a study of acupuncture analgesia and about the theory of acupuncture in traditional Chinese medicine. In reality, a sham acupuncture procedure, involving a retractable needle that does not penetrate the skin, was the only treatment administered. The description of the study in the published article did not explicitly mention deception, although it was implied in the following statement: "Experiments were conducted with the understanding and consent of each subject and approved by the Human Subjects Committee of Massachusetts General Hospital. At the end of the experiment, all subjects were told about the true nature of the experi- ment, because they had been recruited with the understanding that this was a study about acupuncture analgesia." This description suggests that valid consent might have been obtained, although the research deviated from informed consent by being deceptive about the nature of the research and the sham acupuncture intervention. This statement does, however, indicate the use of debriefing - a standard procedure in deceptive research.

Another recently published article presents valuable scientific results concerning the relationship between placebo and nocebo effects and the endogenous opioid and dopaminergic systems (Scott et al., 2008). Healthy volunteers received a muscle pain stimulus, a deceptive placebo intervention designed to elicit placebo and nocebo effects, and brain imaging by means of positron emission tomographic scans. Participants were informed by the investigators that "We are studying the effect of a pain relief medication," and that they would receive either this medication or placebo. In reality, they received only a placebo (saline infusion) in connection with the pain stimulus. Additional deception relating to nocebo effects derived from informing participants about possible adverse effects of the analgesic study medication, which was actually a placebo. In addition to deception regarding the experimental design, it appears that participants were not told that the purpose of the study was to understand placebo and nocebo effects.

The article makes no mention of the use of deception in the experiment. Indeed, the authors give the impression that the study did not involve deception, as they state in the methods section that the disclosure to the participants was similar to typical placebo-controlled trials. In the discussion section, this point is repeated along with enumerating the basic elements of placebocontrolled trials, including "no deception" and "subjects who were aware that either an active or inactive drug could be administered." Unlike placebo-controlled trials, which use concealment of treatment assignment but not deception regarding study interventions, this placebo mechanism experiment deceived the subjects by only administering placebo, despite telling them that they could receive either a pain medication or a placebo.

The methods section also included the statement that "Written informed consent was obtained in all cases." Unless "written informed consent" merely designates the signing of a consent document, informed consent was not obtained. The research involved deception about a key study intervention and probably also about the purpose of the research-two basic elements of informed consent. The article does not mention whether debriefing was used to explain the nature of the deception to the participants after study completion.

A third article describing deceptive experimentation concerning the effects of expectations on appraisal of wine is analogous to the previous articles relating to the placebo effect and further illuminates ethical issues in the design and reporting of neuroscience research (Plassmann et al., 2008). To investigate the effects of the retail price of wine on perceived pleasantness, 20 normal subjects were invited to drink five different Cabernet Sauvignons described as having five different retail prices, ranging from $\$ 5$ to $\$ 90$, while undergoing fMRI. In reality, they drank three different wines, two of which were administered twice- once identified as at a high retail price and once at a low price. In addition they were misinformed that the purpose of the research was "to study the effect of degustation time on perceived flavors." Of the three articles, this was the most transparent about the use of deception. The authors noted that "Although the experiment contains an element of deception, subjects were not debriefed after the experiment to avoid contaminating California Institute of Technology's small subject pool." Whether this is an adequate reason for forgoing debriefing is debatable, especially as the very publication of the study results could itself have the same effect of alerting the subject pool of the use of deception in research at this institution. As in the other articles, the description of the consent process was misleading: "Subjects were informed about the experiment and gave written consent before participating."

In sum, these three articles display varying lack of transparency about the use of experimental deception. Each of them asserted or implied that informed consent was obtained, despite the fact that subjects were deceived about the nature of the research and some of the experimental procedures. In this respect, they reflect common practice in the publication of articles reporting deceptive experiments on the placebo effect (Miller et al., 2005).

Deception relating to the purpose of a study and the procedures used deviates from the required elements of informed consent specified in the United States regulations. As noted above, the regulations permit a waiver or alteration of elements of informed consent when four conditions are satisfied. Accordingly, it is mis- 
leading for articles reporting research that uses deception to include boilerplate statements that informed consent was obtained without explaining whatever deviations or qualifications pertained. Rather, transparency would be served by stating that specific elements of informed consent, such as accurate description of the nature of the study and some of the experimental procedures, were not included in the consent disclosure to participants, and that this deviation from informed consent was approved by an IRB or research ethics committee in accordance with the four regulatory criteria listed above.

\section{Promoting autonomy in deceptive research}

Two measures can be used in deceptive research to minimize infringement of the autonomy of research subjects. First, prospective subjects can be given an "authorized deception" disclosure that alerts them to the use of deception in the research without informing them about the specific nature of that deception (Wendler and Miller, 2004). In an authorized deception disclosure, prospective subjects are informed that some statements about the research are not accurate or misleading; that this use of deception is necessary for the scientific validity of the research and has been approved by a research ethics committee; and that they will receive an accurate account of the research after completing the study. Although use of authorized deception falls short of full informed consent, it promotes the autonomy of research subjects by giving individuals invited to participate in research a fair opportunity to decide whether or not they are willing to volunteer for a study that involves deception.

As a second measure, in the context of debriefing, research participants can be offered the opportunity to withdraw their data from the research after they have learned about the true nature of the study (Sieber, 1983). This procedure restores an element of autonomy to research participants who were deceived during the consent process. It gives them the freedom after the fact to decide whether they wish to contribute to the research now that they are accurately informed. It also provides some assurance that subjects are not contributing to research to which they would have declined to volunteer had they known the nature of the research and the procedures used.

Neither of these procedures to promote the autonomy of participants in deceptive research are novel, but they are not commonly used. Investigators may resist adopting these procedures because of concerns about compromising scientific validity as well as making it more difficult to recruit or retain research subjects or make use of data that they have labored to gather. However, whether authorized deception or permitting withdrawal of data will have adverse effects on research are empirical questions that deserve investigation (Miller et al., 2005). The hypothesis that an authorized deception approach would produce difficulties in recruitment and/or bias subject responses can be tested by conducting ethics substudies attached to deceptive experiments. Subjects can be randomized to an authorized deception disclosure versus a standard consent procedure that does not inform them about the use of deception. Questions to be addressed would include: (1) Do more prospective subjects decline participation in the authorized deception arm? and (2) Are there any significant differences in outcomes between the two groups? In this manner, authorized deception can receive an evidence-based evaluation of its utility as a measure to enhance the ethics of deceptive research without substantially compromising scientific validity. Obviously, such ethics-related research would present serious hurdles because of the need to significantly increase sample size; however, the investment seems warranted to clarify experimentally such empirical issues with critical ethical implications. To assess the impact of permitting subjects to withdraw their data during debriefing, investigators should track and report the extent to which subjects take up the offer.

\section{The role of scientific journals}

The primary responsibility for assuring appropriate ethical standards and transparency in the use of deception in neuroscience rests with investigators and research ethics committees. However, peer reviewers and journal editors play an important role (Miller and Rosenstein, 2002). The lack of transparency described above in published articles that report deceptive experiments can be discerned by careful review of submitted manuscripts. In addition, editors can stipulate reporting standards for deceptive research. These should include the following: explicit description of deceptive aspects of the research; the ways in which the consent process deviated from the elements of informed consent and approval of this alteration of informed consent by a research eth- ics committee; whether the authorized deception approach was used; and the use of debriefing, including whether participants were given the opportunity to withdraw data.

If there are concerns about adding length to published articles, relevant details can be described in supporting documentation available online. Adequate standards for reporting of research involving deception not only can promote transparency but are likely to lead to ethical improvements in the design and review of neuroscience.

\section{References}

Benedetti F, Mayberg HS, Wager TD, Stohler CS Zubieta JK (2005) Neurobiological mechanisms of the placebo effect. J Neurosci 25:10390-10402.

Charron J, Rainville P, Marchand S (2006) Direct comparison of placebo effects on clinical and experimental pain. Clin J Pain 22:204-211.

Department of Health and Human Services (1991) Protection of human subjects. Code of Federal Regulations, 45CFR46.116.(d).

Guess HA, Kleinman A, Kusek JW, Engel LW (2002) The science of the placebo. London: BMJ Books.

Kaptchuk TJ (1998) Powerful placebo: the dark side of the randomised controlled trial. Lancet 351:1722-1725.

Kong J, Gollub RL, Rosman IS, Webb JM, Vangel MG, Kirsch I, Kaptchuk TJ (2006) Brain activity associated with expectancy-enhanced placebo analgesia as measured by functional magnetic resonance imaging. J Neurosci 26:381-388.

Korn JH (1997) Illusions of reality: a history of deception in social psychology. Albany: State University of New York.

Miller FG, Rosenstein DL (2002) Reporting of ethical issues in publications of medical research. Lancet 360:1326-1328.

Miller FG, Wendler D, Swartzman L (2005) Deception in research on the placebo effect. PLoS Med 2:e262.

National Commission for the Protection of $\mathrm{Hu}$ man Subjects of Biomedical and Behavioral Research (1979) The Belmont report. Washington, DC: U.S. Government Printing Office.

Plassmann H, O'Doherty J, Shiv G, Rangel A (2008) Marketing actions can modulate neural representations of experienced pleasantness. Proc Natl Acad Sci USA 105:1050-1054.

Scott DJ, Stohler CS, Egnatuk CM, Wange H, Koeppe RA, Zubieta JK (2008) Placebo and nocebo effects are defined by opposite opioid and dopaminergic responses. Arch Gen Psychiatry 65:220-231.

Sieber JE (1983) Deception in social research III: the nature and limits of debriefing. IRB 5:1-6.

Vase I, RileyJ, Price D (2002) A comparison of placebo effects in clinical analgesic trials versus studies of placebo analgesia. Pain 99:443-452.

Wendler D, Miller FG (2004) Deception in pursuit of science. Arch Intern Med 164:597-600. 